\title{
OPEN Rate of decline in kidney function and known age-of-onset or duration of type 2 diabetes
}

\author{
Oyunchimeg Buyadaa ${ }^{1,2}$, Agus Salim ${ }^{3,4,5,6}$, Jedidiah I. Morton ${ }^{1,2}$, Dianna J. Magliano ${ }^{1,2,7}$ \&
} Jonathan E. Shaw ${ }^{1,2,7}$

The association between rate of kidney function decline and age-of-onset or duration of diabetes has not been well investigated. We aimed to examine whether rates of estimated glomerular filtration rate (eGFR) decline differ by age-of-onset or duration in people with type 2 diabetes. Using the Action to Control Cardiovascular Risk in Diabetes study which included those with $\mathrm{HbA} 1 \mathrm{C} \geq 7.5 \%$ and who were at high risk of cardiovascular events, , rates of eGFR decline were calculated and were compared among groups defined by the known age-of-onset (0-39, 40-49, 50-59, 60-69 and $>70$ years) and 5 -year diabetes duration intervals. Changes in renal function were evaluated using median of 6 (interquartile range 3-10) eGFR measurements per person. eGFR decline was the slowest in those with known age-at-diagnosis of 50-59 years or those with duration of diabetes $<5$ years. The rates of eGFR decline were significantly greater in those with known age-of-onset $<40$ years or those with duration of diabetes $>20$ years compared to those diagnosed at 50-59 or those with duration of diabetes $<5$ years (- $1.98 \mathrm{vs}-1.61 \mathrm{~mL} / \mathrm{min} /$ year; $-1.82 \mathrm{vs}-1.52 \mathrm{~mL} / \mathrm{min} /$ year; respectively $(p<0.001)$. Those with youngest age-of-onset or longer duration of diabetes had more rapid declines in eGFR compared to those diagnosed at middle age or those with shorter duration of diabetes.

Type 2 diabetes diagnosed at a younger age is reported to run an aggressive course and have higher rates of complications compared to older onset type 2 diabetes $^{1-4}$. Indeed, a number of studies have shown that the risk of end-stage kidney disease (ESKD) is higher in younger-onset (i.e., age-of-onset $<40$ years) type 2 diabetes than in older-onset (i.e., age-of-onset $\geq 40$ years) type 2 diabetes, and this excess risk is primarily attributable to attainment of a longer duration of diabetes ${ }^{2,5,6}$. This would suggest that for renal outcomes, younger onset type 2 diabetes is not inherently more aggressive than older onset type 2 diabetes. However, results of these studies of ESKD may be limited, as those younger at onset of diabetes will typically need to have had a greater decline in renal function to reach the ESKD endpoint, as their estimated glomerular filtration rate (eGFR) is typically higher when diabetes is diagnosed compared to their older counterparts. Thus, similar incidence of ESKD may co-exist with different rates of decline in renal function. To gain further understanding of any differences in pathophysiology between younger and older onset or those with shorter and longer duration of type 2 diabetes, analyses of changes in kidney function over time should also be examined. The aim of this study, therefore, was to examine whether rates of eGFR decline differ by known age-of-onset or duration in people with type 2 diabetes. The Action to Control Cardiovascular Risk in Diabetes (ACCORD) clinical trial dataset provides a large sample size with multiple measures of eGFR over time, allowing this aim to be comprehensively explored.

\section{Methods}

Study design and participants. Detailed information about the ACCORD study is described elsewhere ${ }^{7}$. In brief, ACCORD was a multicentre randomised clinical trial in the US and Canada, comprising 10,251 people with type 2 diabetes, glycated haemoglobin levels (HbA1c) of 7.5\% or more, aged $40-79$ with a history of cardiovascular disease (CVD) or the presence of risk factors for CVD. ACCORD recruited participants between 2003

\footnotetext{
${ }^{1}$ Department of Clinical Diabetes and Epidemiology, Baker Heart and Diabetes Institute, 99 Commercial Road, Melbourne, VIC 3004, Australia. ' ${ }^{S}$ chool of Public Health and Preventive Medicine, Monash University, Melbourne, Australia. ${ }^{3}$ Baker Heart and Diabetes Institute, Melbourne, Australia. ${ }^{4}$ Centre for Epidemiology and Biostatistics, Melbourne School of Population and Global Health, The University of Melbourne, Melbourne, Australia. ${ }^{5}$ School of Mathematics and Statistics, The University of Melbourne, Melbourne, Australia. ${ }^{6}$ Department of Mathematics and Biostatistics, La Trobe University, Melbourne, Australia. ${ }^{7}$ These authors jointly supervised this work: Dianna J. Magliano and Jonathan E. Shaw. ${ }^{\square}$ email: Oyunchimeg.Buyadaa@baker.edu.au
} 
and 2005. People with type 1 diabetes were excluded via history and clinical assessment. The passive ACCORD Follow-On (ACCORDION) study involved observation of those members of the ACCORD population who agreed to participate for long-term follow-up. The ACCORD study was approved by the Institutional Review Boards of each study centres and adhered to the principles of the Declaration of Helsinki. All study participants provided written informed consent forms ${ }^{8}$. For the current analysis, we included 9917 participants after excluding those with missing diabetes duration $(n=92)$, missing baseline eGFR $(n=59)$ or absence of any eGFR measurements after baseline $(\mathrm{n}=183)$.

Demographic and clinical variables. Socio-demographics, medical history, concomitant medication use, lifestyle behaviours, health-related quality of life, measures of physical and clinical examinations incorporating kidney function were recorded with different frequency by treatment group assignment as described previously ${ }^{9}$. Medical history including diabetes related data are collected and documented at baseline in the form of a detailed initial medical history and reviewed and recorded at specified follow-up visits.

Serum creatinine was measured at baseline and every 4 months thereafter until the end of the trial and at least once during the post-trial period. The Chronic Kidney Disease Epidemiology Collaboration (CKD-EPI) equation was used to estimate $\mathrm{GFR}^{10}$. Detailed information about measurement methods for laboratory parameters is available in previous reports ${ }^{7,11}$.

Outcome assessment. In this analysis, we specifically focused on the progression of chronic kidney disease (CKD), and therefore evaluated the annual absolute and percentage changes in eGFR. Rates of eGFR decline were calculated and compared based on the known age-of-onset of diabetes, which was classified into 5 groups: $0-39,40-49,50-59,60-69$ and over 70 years and 5-year duration of diabetes intervals ${ }^{5,12}$. ESKD during the trial period is defined as a need for dialysis or renal transplantation or decline of eGFR $\leq 15 \mathrm{~mL} / \mathrm{min} / 1.73 \mathrm{~m}^{2}$ in the absence of an acute reversible cause. ESKD events during the post-trial period in ACCORDION were not captured. Thus, for the post-trial period, we defined ESKD as progression to a sustained eGFR $\leq 15 \mathrm{~mL} / \mathrm{min} / 1.73 \mathrm{~m}^{2}$.

Statistical analyses. Rates of eGFR decline were calculated using a joint longitudinal-survival model and were compared based on the age-of-onset or duration of diabetes ${ }^{13-15}$. In brief, joint-longitudinal survival model is a method that takes into account potentially informative censoring when modelling longitudinal data by simultaneously modelling the longitudinal and survival outcome. This model consists of 2 parts: a model for the trajectory of the longitudinal measurements (eGFR) and a model for the time-to-event (ESKD) data. When longitudinal and event time processes are correlated they reduce the bias obtained from separate a linear mixed effects model or a survival model (time-to-event model). Model 1 included socio-demographic factors; for model 2, smoking status at baseline (yes/no), family history of CVD, personal CVD history, diabetes duration, body mass index, blood pressure levels, use of renin-angiotensin-aldosterone system (RAAS) blockers, glycated haemoglobin, serum lipid levels and urine albumin/creatinine ratio (UACR) were added to model 1. The trajectories of eGFR stratified by age-of-onset of diabetes over time were visualised using duration as the time scale in the analysis. In a sensitivity analysis, we also stratified the analyses by sex. Moreover, in order to determine if hyperfiltration affected the results, we conducted a sensitivity analysis in which only those with a baseline eGFR of less than $120 \mathrm{~mL} / \mathrm{min} / 1.73 \mathrm{~m}^{2}$ were included, and among whom we estimated rates of eGFR decline using a joint longitudinal-survival model. In a further sensitivity analysis, we restricted the analysis to the trial period in which more frequent creatinine measurements were performed. All statistical analyses were performed in Stata for Windows, version 15 (Stata corporation) and R version 3.6.0 (www.r-project.org).

We received a de-identified dataset from the Biologic Specimen and Data Repository Information Coordinating Center (BioLINCC) after obtaining institutional review board approval from the human research ethics committees of the Alfred Hospital (Project No: 214/18) and Monash University (Project No: 13458), Melbourne, Australia.

Disclaimer. This study does not necessarily reflect the opinions or reviews of the ACCORD study investigators or NHLBI.

\section{Results}

Tables 1 and 2 show that at baseline, the median age was 62.0 years (interquartile range (IQR) 57.6-67.0), the median eGFR was $87.2 \mathrm{~mL} / \mathrm{min} / 1.73 \mathrm{~m}^{2}$, and $35.0 \%$ had a prior CVD history. The median age at baseline ranged from 58 years (interquartile range (IQR) (55.3-62.6)) in those with known age-of-onset under 40 years, to 76 years (IQR 74.1-78.1) in those with known age-of-onset of over 70 years. The median known diabetes duration ranged from 3.0 (IQR 1.0-5.0) in those with known age-of-onset of over 70 years to 23.0 (IQR 19.0-30.0) in those with known age-of-onset under 40 years (Tables 1 and 2). With regard to kidney function, the median eGFR at baseline was $91 \mathrm{~mL} / \mathrm{min} / 1.73 \mathrm{~m}^{2}$ in those with known age-of-onset under 40 years and $71 \mathrm{~mL} / \mathrm{min} / 1.73 \mathrm{~m}^{2}$ in those with known age-of-onset of over 70 years. At baseline, those with known age-of-onset under 40 years or those with known duration of diabetes longer than 20 years had poorer glycaemic control, higher UACR and were more likely to be current smokers. They were more likely to be prescribed RAAS-blockers and insulin compared to other age-of-onset or diabetes duration groups (Table 1). Changes in renal function were evaluated using 108,876 eGFR determinations over 9 years (median: 6 (IQR 3-10) determinations per person). Tables 3 and 4 show the rates of eGFR decline according to known age-of-onset or duration of diabetes. 'When adjusted for baseline age, sex, ethnicity and education (Model 1), those with known age-of-onset of type 2 diabetes under 40 years had a faster decline in eGFR for both absolute and percentage changes compared to those diagnosed at ages 50-59 years. Also, those with known duration of diabetes longer than 20 years had a faster absolute and 


\begin{tabular}{|c|c|c|c|c|c|c|}
\hline \multirow[b]{2}{*}{ Characteristics } & \multirow[b]{2}{*}{ Overall } & \multicolumn{5}{|c|}{ Known age-of-onset of diabetes (years) } \\
\hline & & $0-39$ & $40-49$ & $50-59$ & 60-69 & $70+$ \\
\hline $\mathrm{n}(\%)$ & 9917 & $945(9.5)$ & $2926(29.5)$ & $4264(43)$ & $1561(15.8)$ & $221(2.2)$ \\
\hline \begin{tabular}{|l|}
$\begin{array}{l}\text { Baseline age (yr; } \\
\text { median [IQR]) }\end{array}$ \\
\end{tabular} & $62.0(57.6,67.0)$ & $58.0(55.3,62.6)$ & $58.8(56.1,62.8)$ & $61.8(58.4,65.7)$ & $69.1(66.1,72.4)$ & $76.2(74.1,78.1)$ \\
\hline \begin{tabular}{|l|}
$\begin{array}{l}\text { Diabetes duration (yr; } \\
\text { median [IQR]) }\end{array}$ \\
\end{tabular} & $9.0(5.0,15.0)$ & $23.0(19.0,30.0)$ & $13.0(10.0,18.0)$ & $7.0(4.0,11.0)$ & $5.0(3.0,8.0)$ & $3.0(1.0,5.0)$ \\
\hline $\begin{array}{l}\text { Follow-up (yr, mean } \\
{[\mathrm{SD}] \text { ) }}\end{array}$ & $7.0(2.7)$ & $6.9(3.1)$ & $7.0(3.1)$ & $7.1(3.1)$ & $6.6(3.0)$ & $5.5(2.7)$ \\
\hline Male (n [\%]) & $6107(61.6)$ & $542(57.3)$ & $1815(62.0)$ & $2633(61.7)$ & $983(63.0)$ & $136(61.5)$ \\
\hline \multicolumn{7}{|l|}{ Race (n [\%]) } \\
\hline White & $6191(62.4)$ & $504(53.3)$ & $1736(59.3)$ & $2714(63.7)$ & $1083(69.4)$ & $154(69.7)$ \\
\hline Black & $1883(19.0)$ & $217(23.0)$ & $595(20.3)$ & $773(18.1)$ & $261(16.7)$ & 37 (16.7) \\
\hline Hispanic & $709(7.2)$ & $97(10.3)$ & $216(7.4)$ & $311(7.3)$ & $72(4.6)$ & $13(5.9)$ \\
\hline Other & $1134(11.4)$ & $127(13.4)$ & $379(13.0)$ & $466(10.9)$ & $145(9.3)$ & $17(7.7)$ \\
\hline \multicolumn{7}{|l|}{ Education (n [\%]) } \\
\hline$<$ High school & $1442(14.5)$ & $147(15.5)$ & 391 (13.4) & $588(13.8)$ & $270(17.3)$ & $46(20.8)$ \\
\hline High school graduate & $2620(26.4)$ & $266(28.2)$ & $722(24.7)$ & $1102(25.8)$ & $466(29.8)$ & 64 (28.9) \\
\hline Some college & $3257(32.9)$ & $312(33.1)$ & $1016(34.7)$ & $1406(33.0)$ & $463(29.7)$ & $60(27.2)$ \\
\hline$\geq$ College graduate & $2598(26.2)$ & $220(23.2)$ & $797(27.2)$ & $1167(27.4)$ & $362(23.2)$ & $51(23.1)$ \\
\hline \begin{tabular}{|l|}
$\begin{array}{l}\text { Current smoker (n } \\
[\%])\end{array}$ \\
\end{tabular} & $1375(13.9)$ & $145(15.3)$ & $451(15.4)$ & $594(13.9)$ & $169(10.8)$ & $16(7.2)$ \\
\hline $\begin{array}{l}\text { HbAlc (\%; median } \\
\text { [IQR]) }\end{array}$ & $8.1(7.6,8.9)$ & $8.3(7.8,9.1)$ & $8.2(7.7,9.0)$ & $8.1(7.5,8.8)$ & $7.9(7.5,8.6)$ & $7.8(7.3,8.6)$ \\
\hline \begin{tabular}{|l|}
$\mathrm{HbAlc}(\mathrm{mmol} / \mathrm{mol} ;$ \\
median [IQR])
\end{tabular} & $65.0(58.5,72.3)$ & $67.2(61.7,75.9)$ & $66.1(60.6,74.9)$ & $65.0(58.5,72.7)$ & $62.8(58.5,70.5)$ & $61.7(56.3,70.5)$ \\
\hline Insulin use (n [\%]) & $3480(35.1)$ & $637(67.4)$ & $1360(46.5)$ & $1161(27.2)$ & $298(19.1)$ & $24(10.8)$ \\
\hline $\begin{array}{l}\text { BMI }\left(\mathrm{kg} / \mathrm{m}^{2} ; \text { mean }\right. \\
[\mathrm{SD}])\end{array}$ & $32.2(5.4)$ & $32.2(5.6)$ & $32.5(5.5)$ & $32.4(5.3)$ & $31.4(4.9)$ & $30.2(4.8)$ \\
\hline $\begin{array}{l}\text { Triglycerides (mg/dL; } \\
\text { median [IQR]) }\end{array}$ & $155(106,228)$ & $137(94.5,202)$ & $151(102,232)$ & $160(112,236)$ & $157(109.5,218.5)$ & $148(99,218)$ \\
\hline $\begin{array}{l}\text { Total cholesterol (mg/ } \\
\text { dL; median }[\mathrm{IQR}])\end{array}$ & $178(154,207)$ & $174(149,201)$ & $177(153,208)$ & $181(157,209)$ & $176(153,203)$ & $171(147,198)$ \\
\hline $\begin{array}{l}\text { Systolic BP (mmHg; } \\
\text { mean }[\mathrm{SD}])\end{array}$ & $136.3(17.0)$ & $136.2(17.1)$ & $136.1(16.9)$ & $135.8(16.8)$ & $137.5(18.0)$ & $139.0(16.5)$ \\
\hline RAAS blockers (n [\%]) & $6858(69.1)$ & $698(73.8)$ & $2102(71.8)$ & $2864(67.2)$ & $1039(66.5)$ & $154(70.1)$ \\
\hline CVD history (n [\%]) & $3472(35.0)$ & $474(50.1)$ & $1113(38.0)$ & $1241(29.1)$ & $554(35.5)$ & $90(40.9)$ \\
\hline $\begin{array}{l}\text { UACR }(\mathrm{mg} / \mathrm{g} ; \text { median } \\
[\mathrm{IQR}])\end{array}$ & $14(7,44)$ & $20(8,86.5)$ & $14(7,50)$ & $13(7,37)$ & $12(7,37)$ & $17(7,43)$ \\
\hline \multicolumn{7}{|l|}{\begin{tabular}{|l|} 
UACR categories \\
\end{tabular}} \\
\hline $\begin{array}{l}\text { A1 (normoalbumi- } \\
\text { nuria) }\end{array}$ & $6801(68.6)$ & $538(56.9)$ & $1949(66.6)$ & $3062(71.8)$ & $1108(71.0)$ & $144(65.2)$ \\
\hline $\begin{array}{l}\text { A2 (microalbumi- } \\
\text { nuria) }\end{array}$ & $2468(24.9)$ & $297(31.4)$ & $743(25.4)$ & $991(23.2)$ & $371(23.8)$ & $66(29.9)$ \\
\hline $\begin{array}{l}\text { A3 (macroalbumi- } \\
\text { nuria) }\end{array}$ & $648(6.5)$ & $110(11.7)$ & $234(8.0)$ & $211(5.0)$ & $82(5.2)$ & $11(4.9)$ \\
\hline $\begin{array}{l}\text { eGFR }(\mathrm{mL} / \\
\mathrm{min} / 1.73 \mathrm{~m}^{2} ; \text { median } \\
[\mathrm{IQR}])\end{array}$ & $87.2(72.2,96.7)$ & $91.2(72.7,99.4)$ & $91.3(75.6,99.1)$ & $88.8(73.1,96.8)$ & $78.6(66.7,90.4)$ & $71.5(58.6,83.0)$ \\
\hline \multicolumn{7}{|c|}{ eGFR categories $\left(\mathrm{mL} / \mathrm{min} / 1.73 \mathrm{~m}^{2}\right)$} \\
\hline G1 (>90) & $4515(45.5)$ & $491(52.0)$ & $1549(52.9)$ & 2034 (47.7) & $422(27.1)$ & $19(8.6)$ \\
\hline G2 (60-90) & $4414(44.5)$ & $362(38.3)$ & $1149(39.3)$ & $1838(43.1)$ & $923(59.1)$ & $142(64.3)$ \\
\hline G3 $(<60)$ & $988(10.0)$ & $92(9.7)$ & $228(7.8)$ & $392(9.2)$ & $216(13.8)$ & $60(27.1)$ \\
\hline
\end{tabular}

Table 1. Baseline characteristics of study participants according to known age-of-onset of diabetes. $I Q R$ interquartile range, $y$ year-olds, $y r$ year, $H b A 1 c$ glycated haemoglobin, $B M I$ body mass index, $B P$ blood pressure, RAAS blockers renin-angiotensin-aldosterone system blockers, CVD history cardiovascular disease history, UACR urine albumin-creatinine ratio, $e G F R$ estimated glomerular filtration rate.

percentage decline in eGFR compared to those with diabetes duration of less than 5 years. Similarly, the incidence of ESKD was significantly higher in the $<40$ age-of-onset group or those with longer diabetes duration (Supplementary Table S1). Those with known age-of-onset over 70 years also had an annual percentage decline 


\begin{tabular}{|c|c|c|c|c|c|c|}
\hline \multirow[b]{2}{*}{ Characteristics } & \multirow[b]{2}{*}{ Overall } & \multicolumn{5}{|c|}{ Known duration of diabetes (years) } \\
\hline & & $0-4$ & $5-9$ & $10-14$ & $15-19$ & $20+$ \\
\hline n (\%) & 9917 & $2114(21.3)$ & $2848(28.7)$ & $2246(22.7)$ & $1311(13.2)$ & $1398(14.1)$ \\
\hline $\begin{array}{l}\text { Baseline age (yr; median } \\
\text { [IQR]) }\end{array}$ & $62.0(57.6,67.0)$ & $60.4(56.8,65.3)$ & $61.4(57.4,66.2)$ & $61.9(57.7,66.8)$ & $62.9(58.5,68.3)$ & $64.6(60.1,70.1)$ \\
\hline $\begin{array}{l}\text { Age at diagnosis of } \\
\text { diabetes (yr; median } \\
\text { [IQR]) }\end{array}$ & $52.2(46.2,58.0)$ & $58.1(54.3,62.7)$ & $54.7(50.8,59.7)$ & $50.2(46.3,55.3)$ & $46.4(42.0,51.4)$ & $40.1(35.0,45.3)$ \\
\hline $\begin{array}{l}\text { Follow-up (yr; mean } \\
\text { [SD]) }\end{array}$ & $6.9(3.1)$ & $6.9(3.1)$ & $7.0(3.1)$ & $7.1(3.1)$ & $7.0(3.0)$ & $6.6(3.1)$ \\
\hline Male (n [\%]) & $6107(61.6)$ & $1294(61.2)$ & $1772(62.2)$ & $1383(61.5)$ & $810(61.8)$ & $850(60.8)$ \\
\hline \multicolumn{7}{|l|}{ Race (n [\%]) } \\
\hline White & $6191(62.4)$ & $1317(62.3)$ & $1868(65.6)$ & $1399(62.3)$ & $797(60.8)$ & $810(57.9)$ \\
\hline Black & $1883(19.0)$ & $257(12.2)$ & $297(10.4)$ & $251(11.2)$ & $166(12.7)$ & $163(11.7)$ \\
\hline Hispanic & $709(7.2)$ & $150(7.1)$ & $178(6.3)$ & $179(7.9)$ & $85(6.4)$ & $117(8.4)$ \\
\hline Other & $1134(11.4)$ & $390(18.4)$ & $505(17.7)$ & 417(18.6) & $263(20.1)$ & $308(22.0)$ \\
\hline \multicolumn{7}{|l|}{ Education (n [\%]) } \\
\hline$<$ High school & $1442(14.5)$ & $274(12.9)$ & $408(14.3)$ & $309(13.8)$ & $184(14.1)$ & $261(18.7)$ \\
\hline High school graduate & $2620(26.4)$ & $533(25.2)$ & $740(26.0)$ & $613(26.6)$ & $349(26.6)$ & $385(27.5)$ \\
\hline Some college & $3257(32.9)$ & $727(34.4)$ & $949(33.3)$ & $709(31.6)$ & $414(31.7)$ & $458(32.8)$ \\
\hline$\geq$ College graduate & $2598(26.2)$ & $580(27.5)$ & $750(26.4)$ & $614(27.3)$ & $361(27.6)$ & $293(21.0)$ \\
\hline Current smoker (n [\%]) & $1375(13.9)$ & $341(16.1)$ & 415 (14.6) & $312(13.9)$ & $151(11.5)$ & $156(11.1)$ \\
\hline $\begin{array}{l}\text { HbAlc (\%; median } \\
\text { [IQR]) }\end{array}$ & $8.1(7.6,8.9)$ & $7.9(7.4,8.7)$ & $8.1(7.6,8.9)$ & $8.2(7.6,8.9)$ & $8.1(7.6,8.8)$ & $8.2(7.7,8.9)$ \\
\hline $\begin{array}{l}\mathrm{HbAlc}(\mathrm{mmol} / \mathrm{mol} ; \\
\text { median [IQR]) }\end{array}$ & $65.0(58.5,72.3)$ & $62.8(57.3,71.5)$ & $65.0(59.5,73.7)$ & $66.1(59.5,72.6)$ & $65.0(59.5,72.6)$ & $66.1(60.6,73.7)$ \\
\hline Insulin use (n [\%]) & $3480(35.1)$ & $225(10.6)$ & $690(24.2)$ & $908(40.4)$ & $740(56.4)$ & $917(65.6)$ \\
\hline BMI $\left(\mathrm{kg} / \mathrm{m}^{2} ;\right.$ mean $\left.[\mathrm{SD}]\right)$ & $32.2(5.4)$ & $32.6(5.4)$ & $32.4(5.3)$ & $32.2(5.3)$ & $31.8(5.4)$ & $31.6(5.5)$ \\
\hline $\begin{array}{l}\text { Triglycerides (mg/dL; } \\
\text { median [IQR]) }\end{array}$ & $155(106,228)$ & $166(118,246)$ & $165(114,237)$ & $152(105,229)$ & $137(96,205)$ & $133(94,199)$ \\
\hline $\begin{array}{l}\text { Total cholesterol (mg/ } \\
\text { dL; median }[\mathrm{QR}])\end{array}$ & $178(154,207)$ & $185(159,213)$ & $179(156,208)$ & $177(154,207)$ & $174(151,202)$ & $171(149,199)$ \\
\hline $\begin{array}{l}\text { Systolic BP (mmHg; } \\
\text { mean [SD]) }\end{array}$ & $136.3(17.0)$ & $135.5(16.2)$ & $135.1(16.9)$ & $136.5(17.4)$ & $137.6(17.4)$ & $138.0(17.4)$ \\
\hline RAAS blockers (n [\%]) & $6858(69.1)$ & $1299(61.5)$ & $1954(68.6)$ & $1592(70.8)$ & $976(74.4)$ & $1037(74.2)$ \\
\hline CVD history (n [\%]) & $3472(35.0)$ & $690(32.6)$ & $898(31.5)$ & $764(34.0)$ & $496(37.8)$ & $624(44.6)$ \\
\hline $\begin{array}{l}\text { UACR (mg/g; median } \\
\text { [IQR]) }\end{array}$ & $14(7,44)$ & $11(6,26)$ & $12(6,34)$ & $15(7,50)$ & $17(7,59)$ & $22(9,91)$ \\
\hline \multicolumn{7}{|l|}{ UACR categories } \\
\hline A1 (normoalbuminuria) & $6801(68.6)$ & $1631(77.2)$ & $2075(72.8)$ & $1495(66.6)$ & $823(62.8)$ & 777 (55.6) \\
\hline A2 (microalbuminuria) & $2468(24.9)$ & $414(19.6)$ & $632(22.2)$ & $591(26.3)$ & $376(28.7)$ & $455(32.6)$ \\
\hline A3 (macroalbuminuria) & $648(6.5)$ & $69(3.2)$ & $141(5.0)$ & $159(7.1)$ & $112(8.5)$ & $166(11.8)$ \\
\hline $\begin{array}{l}\text { eGFR }\left(\mathrm{mL} / \mathrm{min} / 1.73 \mathrm{~m}^{2} \text {; }\right. \\
\text { median }[\mathrm{IQR}])\end{array}$ & $87.2(72.2,96.7)$ & $91.2(77.7,99.1)$ & $88.7(73.5,97.4)$ & $86.7(72.1,96.7)$ & $83.7(69.5,94.8)$ & $79.6(65.2,93.3)$ \\
\hline \multicolumn{7}{|c|}{ eGFR categories $\left(\mathrm{mL} / \mathrm{min} / 1.73 \mathrm{~m}^{2}\right)$} \\
\hline G1 (>90) & 4515 (45.5) & $1134(53.6)$ & $1356(47.7)$ & $1026(45.7)$ & $528(40.3)$ & $471(33.7)$ \\
\hline G2 (60-90) & $4414(44.5)$ & $852(40.3)$ & $1237(43.4)$ & $1103(45.1)$ & $618(41.1)$ & $694(49.6)$ \\
\hline G3 $(<60)$ & $988(10.0)$ & $128(6.1)$ & $255(8.9)$ & $207(9.2)$ & $165(12.6)$ & $233(16.7)$ \\
\hline
\end{tabular}

Table 2. Baseline characteristics of study participants according to known duration of diabetes. IQR interquartile range, $y$ year-olds, $y r$ year, $H b A 1 c$ glycated haemoglobin, $B M I$ body mass index, $B P$ blood pressure, RAAS blockers renin-angiotensin-aldosterone system blockers, CVD history cardiovascular disease history, UACR urine albumin-creatinine ratio, eGFR estimated glomerular filtration rate.

in eGFR that was significantly greater than the reference group $(-2.75 \mathrm{vs}-1.99, \mathrm{p}<0.001)$. Results for Model 2 were similar to those for Model 1.

Figure 1 shows the trajectories of eGFR stratified by age-of-onset of diabetes. Ten years after diagnosis, people with known age-of-onset of diabetes under 40 years had the highest average eGFR. However, they experience the most rapid decline in kidney function compared to those diagnosed later in life. Over a 10-year period (e.g. from 15 to 25 years duration), their average eGFR declines by $10 \mathrm{~mL} / \mathrm{min} / 1.73 \mathrm{~m}^{2}$ [95\% confidence interval 8.0-12.1] more than does the eGFR of those diagnosed at age 50-59 years. 


\begin{tabular}{|c|c|c|c|c|c|}
\hline & $\mathbf{N}$ & $\begin{array}{l}\text { Absolute eGFR change }(\mathrm{mL} / \\
\left.\mathrm{min} / 1.73 \mathrm{~m}^{2} \text { per } \mathrm{yr}\right)^{\mathrm{a}}\end{array}$ & $\begin{array}{l}\text { Difference in absolute eGFR change } \\
(95 \% \mathrm{CI})\end{array}$ & Percentage eGFR change $\left(\%\right.$ per yr) ${ }^{a}$ & $\begin{array}{l}\text { Difference in percentage eGFR } \\
\text { change }(95 \% \mathrm{CI})\end{array}$ \\
\hline \multicolumn{6}{|l|}{ Model 1} \\
\hline $0-39 y$ & 945 & $-2.01(2.45)$ & $-0.38(-0.58,-0.17)$ & $-2.46(2.64)$ & $-0.47(-0.65,-0.29)$ \\
\hline $40-49 y$ & 2926 & $-1.78(2.04)$ & $-0.15(-0.26,-0.02)$ & $-2.13(2.06)$ & $-0.12(-0.22,-0.03)$ \\
\hline $50-59 y$ & 4264 & $-1.63(1.88)$ & Reference & $-1.99(1.89)$ & Reference \\
\hline $60-69$ y & 1561 & $-1.68(2.35)$ & $-0.05(-0.18,0.08)$ & $-2.21(2.00)$ & $-0.22(-0.33,-0.10)$ \\
\hline$\geq 70 y$ & 221 & $-1.89(2.17)$ & $-0.26(-0.61,0.10)$ & $-2.75(2.28)$ & $-0.76(-1.06,-0.44)$ \\
\hline \multicolumn{6}{|l|}{ Model 2} \\
\hline $0-39 y$ & 943 & $-1.98(2.43)$ & $-0.37(-0.56,-0.16)$ & $-2.35(2.61)$ & $-0.40(-0.57,-0.22)$ \\
\hline $40-49 y$ & 2925 & $-1.75(2.06)$ & $-0.14(-0.25,-0.02)$ & $-2.05(2.25)$ & $-0.10(-0.180 .01)$ \\
\hline $50-59$ y & 4261 & $-1.61(1.89)$ & Reference & $-1.95(1.94)$ & Reference \\
\hline $60-69 y$ & 1559 & $-1.62(1.88)$ & $-0.01(-0.14,0.13)$ & $-2.06(2.08)$ & $-0.11(-0.22,0.01)$ \\
\hline$\geq 70 y$ & 220 & $-1.77(2.21)$ & $-0.16(-0.53,0.20)$ & $-2.52(2.23)$ & $-0.56(-0.91,-0.21)$ \\
\hline
\end{tabular}

Table 3. Annual change in eGFR according to known age-of-onset of diabetes. Model 1: adjusted for age, sex, race and education. Model 2: Model $1+$ smoking status at baseline, family history of cardiovascular disease (CVD), CVD history at baseline, diabetes duration, body mass index, blood pressure levels, use of reninangiotensin-aldosterone system blockers, glycated haemoglobin level, serum lipid levels, and baseline urine albumin/creatinine, ratio. Baseline eGFR is not included in the covariate set, as it is already present in the joint longitudinal-survival model specification. $e$ GFR estimated glomerular filtration rate, $y$ years. ${ }^{a} D a t a$ are mean (standard deviation).

\begin{tabular}{|c|c|c|c|c|c|}
\hline & $\mathbf{N}$ & $\begin{array}{l}\text { Absolute eGFR change }(\mathrm{mL} / \mathrm{min} / 1.73 \\
\left.\mathrm{m}^{2} \text { per } \mathrm{yr}\right)^{\mathrm{a}}\end{array}$ & $\begin{array}{l}\text { Difference in absolute eGFR change } \\
(95 \% \mathrm{CI})\end{array}$ & Percentage eGFR change (\% per yr) ${ }^{a}$ & $\begin{array}{l}\text { Difference in percentage eGFR } \\
\text { change }(95 \% \mathrm{CI})\end{array}$ \\
\hline \multicolumn{6}{|l|}{ Model 1} \\
\hline $0-4 y$ & 2114 & $-1.55(1.87)$ & Reference & $-1.83(1.81)$ & Reference \\
\hline $5-9 y$ & 2848 & $-1.65(1.94)$ & $-0.10(-0.23,0.03)$ & $-2.00(1.94)$ & $-0.17(-0.28,-0.07)$ \\
\hline $10-14 \mathrm{y}$ & 2246 & $-1.80(1.91)$ & $-0.25(-0.39,-0.11)$ & $-2.20(1.93)$ & $-0.37(-0.49,-0.26)$ \\
\hline $15-19 y$ & 1311 & $-1.82(2.18)$ & $-0.27(-0.44,-0.09)$ & $-2.31(2.35)$ & $-0.48(-0.62,-0.33)$ \\
\hline$\geq 20 \mathrm{y}$ & 1398 & $-1.90(2.17)$ & $-0.35(-0.52,-0.17)$ & $-2.53(2.41)$ & $-0.70(-0.85,-0.55)$ \\
\hline \multicolumn{6}{|l|}{ Model 2} \\
\hline $0-4 y$ & 2110 & $-1.52(1.81)$ & Reference & $-1.82(1.74)$ & Reference \\
\hline $5-9 y$ & 2832 & $-1.62(1.89)$ & $-0.10(-0.22,0.04)$ & $-1.91(1.89)$ & $-0.09(-0.19,0.01)$ \\
\hline $10-14 \mathrm{y}$ & 2236 & $-1.77(1.92)$ & $-0.25(-0.38,-0.11)$ & $-2.16(1.95)$ & $-0.34(-0.45,-0.23)$ \\
\hline $15-19 y$ & 1307 & $-1.76(2.14)$ & $-0.40(-0.40,-0.06)$ & $-2.21(2.31)$ & $-0.39(-0.54,-0.25)$ \\
\hline$\geq 20 \mathrm{y}$ & 1393 & $-1.82(2.17)$ & $-0.30(-0.46,-0.12)$ & $-2.40(2.39)$ & $-0.58(-0.72,-0.43)$ \\
\hline
\end{tabular}

Table 4. Annual change in eGFR according to known duration of diabetes. Model 1: adjusted for age, sex, race and education. Model 2: Model $1+$ smoking status at baseline, family history of cardiovascular disease (CVD), CVD history at baseline, diabetes duration, body mass index, blood pressure levels, use of reninangiotensin-aldosterone system blockers, glycated haemoglobin level, serum lipid levels, and baseline urine albumin/creatinine, ratio. Baseline eGFR is not included in the covariate set, as it is already present in the joint longitudinal-survival model specification. $e$ GFR estimated glomerular filtration rate, $y$ years. ${ }^{a}$ Data are mean (standard deviation).

Sensitivity analyses. In a separate analysis, we compared the rate of decline in kidney function according to known age-of-onset or duration of diabetes, stratified by sex. The patterns of the rate of eGFR decline were similar to that of our main analysis (Supplementary Table S2). Both absolute and percentage changes in eGFR were similar to the main results when we excluded those with eGFR $>120 \mathrm{~mL} / \mathrm{min} / 1.73 \mathrm{~m}^{2}$ (Supplementary Table S3). Results were similar in another sensitivity analysis restricted to the trial period, except that those with known age-of-onset over 70 years had a lower annual absolute, but not percentage, decline in eGFR compared to the reference group (Supplementary Table S4).

\section{Discussion}

Using data from this prospective cohort study, we showed that rates of eGFR decline are faster in the youngest age of type 2 diabetes onset group and in those with longest duration of diabetes compared to those with onset aged 50-59 years or those with duration of diabetes less than 5 years, respectively. We further showed that in 


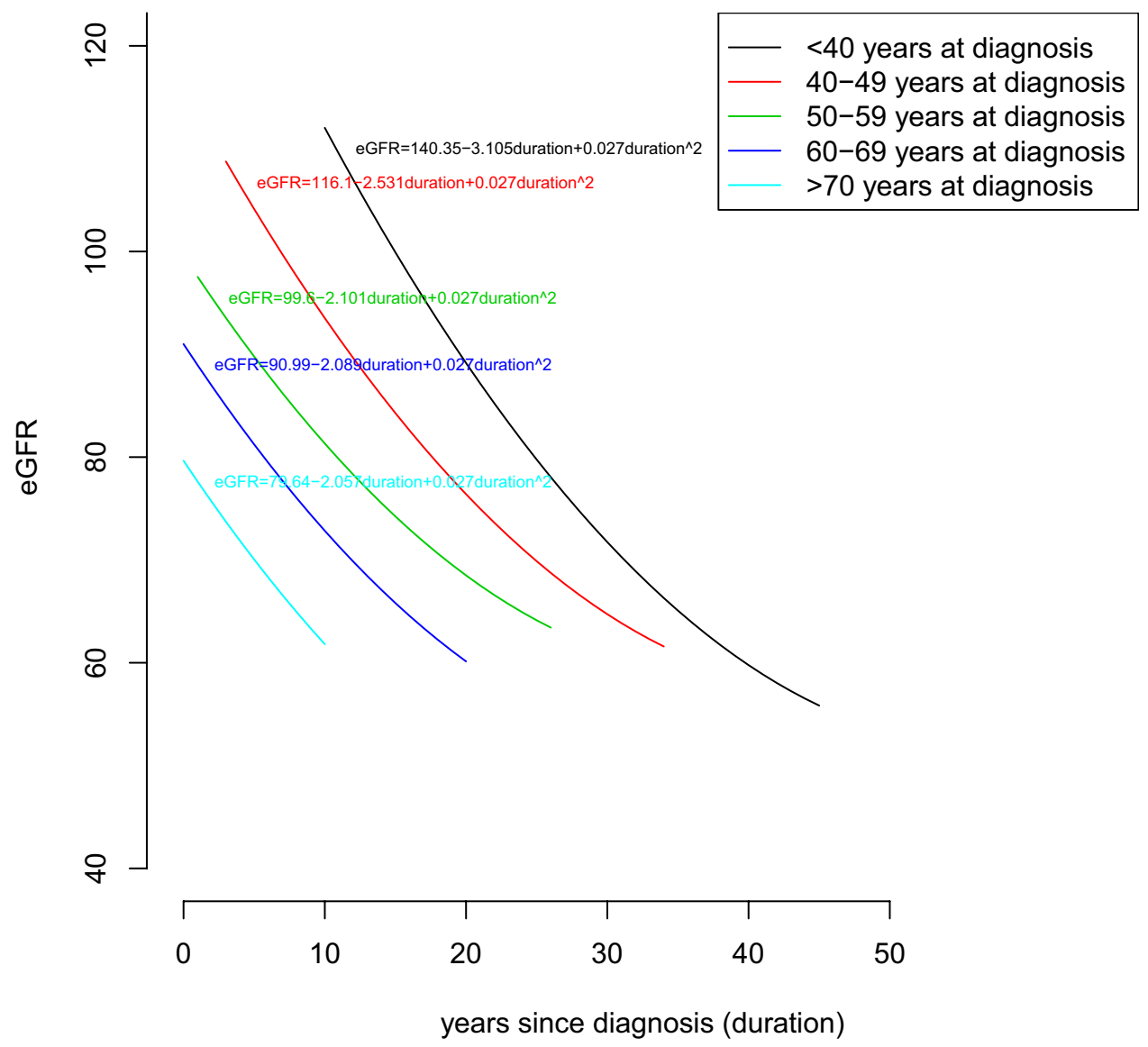

Figure 1. Estimated trajectories of log-transformed estimated glomerular filtration rate (eGFR) levels according to known diabetes duration based on Model 1, stratified by known age-of-onset of diabetes.

this cohort of people with type 2 diabetes, among those with similar but longer diabetes durations, the rate of eGFR decline is greatest in those with earliest diabetes onset.

The baseline clinical characteristics of study participants with younger onset (those with known age-ofonset $<40$ years) or longer duration (those with known diabetes duration $>20$ years) of type 2 diabetes in this study were similar to those reported in other studies ${ }^{16,17}$. The younger diabetes onset or those with longer duration group had worse glycaemic control and were more likely to be current smokers. These unfavourable risk factors may partly contribute to a more aggressive progression of diabetes and higher rates of complications. In addition, the prevalence of albuminuria was higher in those with younger-onset or longer duration of diabetes compared to any other group. These results suggest that albuminuria could be more relevant for kidney dysfunction in type 2 diabetes. This is in keeping our previous analysis of the ACCORD cohort ${ }^{15}$.

While there is good evidence showing that younger onset type 2 diabetes confers an increased risk of ESKD ${ }^{2,4}$, a finding we confirmed here, these studies do not report on rates of eGFR decline by age-of-onset or duration of diabetes. Thus, this may not represent differences in pathophysiology of CKD progression between younger and older onset type 2 diabetes or how pathophysiology changes with increasing diabetes duration. Clearly, individuals who develop diabetes at a younger age will need to have had a greater decline in renal function to reach ESKD, because their initial eGFR is typically higher when diabetes is diagnosed ${ }^{2,16,18}$. Examination of the rate of decline in eGFR therefore provides important additional insights into the impact of younger onset or longer duration of type 2 diabetes. Our current analyses of changes in eGFR over time demonstrated that both the mean absolute and percentage annual declines in eGFR in people with younger onset type 2 diabetes appears to be greater than those diagnosed in middle age, and that the annual eGFR decline increases with increasing duration of diabetes. These findings suggest that the pathophysiological mechanisms of CKD progression in younger onset diabetes may be different to older onset diabetes; and that known age-of-onset and duration of diabetes are both important factors underlying the progression of CKD in diabetes.

There are several possible explanations for our findings. One possible explanation for rapid decline in eGFR in people with younger onset (those with known age-of-onset $<40$ years) type 2 diabetes, is the hyperfiltration and its subsequent normalisation in this group, which may lead to greater decline in $\mathrm{GFFR}^{8,19}$. However, this explanation is unlikely, because differences in eGFR decline persisted after excluding those with possible hyperfiltration in our sensitivity analysis. It is possible that some features of obesity, including increased levels of fatty acids and leptin, may contribute to greater decline in kidney function in those who develop diabetes at younger age ${ }^{20,21}$. The elevated levels of fatty acids is accompanied by increased oxidative stress which, together may result in 
premature kidney damage ${ }^{22}$. The biomarkers associated with progression of kidney disease may also change with increasing diabetes duration. Furthermore, genetic predisposition and other unknown factors associated with a younger onset of type 2 diabetes may play a role in the more rapid decline in kidney function in this group ${ }^{23,24}$.

Although people with an age-of-onset over 70 years seemed to have a greater decline in eGFR compared to the reference group in the primary analysis, when we restricted the analysis to the ACCORD trial period, this was no longer statistically significant. It is not clear why this only affected the oldest age-of-onset group. It is possible that the intensive therapy in the in-trial period was relaxed more quickly at the end of ACCORD in this group than in younger participants. Thus, eGFR started to fall more rapidly in the ACCORDION phase.

The strengths of this study are the large sample size, long follow-up and frequent serum creatinine sampling. However, our data should be interpreted carefully in the context of the following limitations. Ascertaining the exact age-of-onset in type 2 diabetes is difficult, because many people remain asymptomatic or undiagnosed for many years. In addition, we were unable to completely account for differences in duration of diabetes between age-of-onset groups. This is because age-of-onset and duration of diabetes are highly correlated, and because of the limited overlap of durations in the younger age-of-onset groups with older age-of-onset. Moreover, what we found mainly applies to people aged over 50 years old, and cannot necessarily be extrapolated to the earlier years of diabetes in those with younger onset type 2 diabetes. Lastly, the generalizability of our findings may be limited to some extent, because the population was drawn from a clinical trial that recruited those with HbAlc $\geq 7.5 \%$ and who were at high risk of CVD events. However, we believe that this cohort represents a diverse group of people with type 2 diabetes and our findings add significantly to the understanding of potential differences in progression of CKD for those with a younger onset of type 2 diabetes.

\section{Conclusion}

Our current study suggests that in cohort of people type 2 diabetes with $\mathrm{HbAlc} \geq 7.5 \%$ and who were at high risk of CVD events, those with younger age-of-onset or longer duration of type 2 diabetes may have a more rapid decline in eGFR compared to those diagnosed in middle age or those with shorter duration of diabetes. These findings contribute to the body of evidence suggesting that early and careful monitoring of kidney function is warranted in those with younger onset type 2 diabetes as they are at the highest long-term risk for kidney complications. Interventions which halt or slow the decline of eGFR are needed in this group.

Received: 2 March 2021; Accepted: 29 June 2021

Published online: 19 July 2021

\section{References}

1. Lascar, N. et al. Type 2 diabetes in adolescents and young adults. Lancet Diabetes Endocrinol. 6, 69-80 (2018).

2. Pavkov, M. E. et al. Effect of youth-onset type 2 diabetes mellitus on incidence of end-stage renal disease and mortality in young and middle-aged Pima Indians. JAMA 296, 421-426 (2006).

3. Hillier, T. A. \& Pedula, K. L. Complications in young adults with early-onset type 2 diabetes: Losing the relative protection of youth. Diabetes Care 26, 2999-3005 (2003).

4. Magliano, D. J. et al. Young-onset type 2 diabetes mellitus-Implications for morbidity and mortality. Nat. Rev. Endocrinol. 16, $1-11(2020)$.

5. Chan, J. C. et al. Premature mortality and comorbidities in young-onset diabetes: A 7-year prospective analysis. Am. J. Med. 127, 616-624 (2014).

6. Morton, J. I., Liew, D., McDonald, S. P., Shaw, J. E. \& Magliano, D. J. The association between age of onset of type 2 diabetes and the long-term risk of end-stage kidney disease: A national registry study. Diabetes Care 43, 1788-1795 (2020).

7. Buse, J. B. \& Group, A. S. Action to Control Cardiovascular Risk in Diabetes (ACCORD) trial: Design and methods. Am. J. Cardiol. 99, S21-S33 (2007).

8. Ismail-Beigi, F. et al. Effect of intensive treatment of hyperglycaemia on microvascular outcomes in type 2 diabetes: An analysis of the ACCORD randomised trial. Lancet 376, 419-430 (2010).

9. ACCORD Study Group. Nine-year effects of 3.7 years of intensive glycemic control on cardiovascular outcomes. Diabetes Care 39, 701-708 (2016).

10. Levey, A. S. \& Stevens, L. A. Estimating GFR using the CKD epidemiology collaboration (CKD-EPI) creatinine equation: More accurate GFR estimates, lower CKD prevalence estimates, and better risk predictions. Am. J. Kidney Dis. 55, 622-627 (2010).

11. Bonds, D. et al. Fenofibrate-associated changes in renal function and relationship to clinical outcomes among individuals with type 2 diabetes: The Action to Control Cardiovascular Risk in Diabetes (ACCORD) experience. Diabetologia 55, 1641-1650 (2012).

12. Li, L. et al. Prevalence of microvascular diseases among tertiary care Chinese with early versus late onset of type 2 diabetes. $J$. Diabetes Complicat. 29, 32-37 (2015).

13. Henderson, R., Diggle, P. \& Dobson, A. Joint modelling of longitudinal measurements and event time data. Biostatistics 1, 465-480 (2000).

14. Asar, Ö., Ritchie, J., Kalra, P. A. \& Diggle, P. J. Joint modelling of repeated measurement and time-to-event data: An introductory tutorial. Int. J. Epidemiol. 44, 334-344 (2015).

15. Buyadaa, O., Magliano, D. J., Salim, A., Koye, D. N. \& Shaw, J. E. Risk of rapid kidney function decline, all-cause mortality, and major cardiovascular events in nonalbuminuric chronic kidney disease in type 2 diabetes. Diabetes Care 43, 122-129 (2020).

16. Liu, J.-J. et al. Risk of progressive chronic kidney disease in individuals with early-onset type 2 diabetes: A prospective cohort study. Nephrol. Dial. Transplant. 35, 115-121 (2020).

17. Yeung, R. O. et al. Metabolic profiles and treatment gaps in young-onset type 2 diabetes in Asia (the JADE programme): A crosssectional study of a prospective cohort. Lancet Diabetes Endocrinol. 2, 935-943 (2014).

18. Thomas, M. C., Cooper, M. E. \& Zimmet, P. Changing epidemiology of type 2 diabetes mellitus and associated chronic kidney disease. Nat. Rev. Nephrol. 12, 73 (2016).

19. Ruggenenti, P. et al. Glomerular hyperfiltration and renal disease progression in type 2 diabetes. Diabetes Care 35, 2061-2068 (2012).

20. Lin, J., Hu, F. B., Mantzoros, C. \& Curhan, G. C. Lipid and inflammatory biomarkers and kidney function decline in type 2 diabetes. Diabetologia 53, 263 (2010). 
21. Bjornstad, P. et al. Insulin sensitivity and diabetic kidney disease in children and adolescents with type 2 diabetes: An observational analysis of data from the TODAY clinical trial. Am. J. Kidney Dis. 71, 65-74 (2018).

22. Prkačin, I. Multiple obesity related mechanisms of kidney disease. Cardiol. Croatica 12, 315-318 (2017).

23. Maeda, S. et al. Genetic variations associated with diabetic nephropathy and type II diabetes in a Japanese population. Kidney Int. 72, S43-S48 (2007).

24. Rich, S. S. Genetics of diabetes and its complications. J. Am. Soc. Nephrol. 17, 353-360 (2006).

\title{
Acknowledgements
}

The authors thank the investigators, staff, and participants of the ACCORD study for their contributions and for giving us access to this rich and valuable dataset. The ACCORD study was conducted by the ACCORD study investigators and supported the National Heart, Lung, and Blood Institute (NHLBI); by other components of the National Institutes of Health, including the National Institute of Diabetes and Digestive and Kidney Diseases, the National Institute on Aging, and the National Eye Institute; by the Centers for Disease Control and Prevention; and by General Clinical Research Centers.

\section{Author contributions}

O.B., D.J.M., J.I.M. and J.E.S. designed the study. O.B. and A.S. performed data analysis and interpreted the results. O.B. wrote the manuscript. D.J.M., A.S. and J.E.S. supervised the application of the joint longitudinalsurvival model and reviewed and edited the manuscript. O.B., D.J.M. and J.E.S. are the guarantors of this work and, as such, had full access to all the data in the study and take responsibility for the integrity of the data and accuracy of the data analysis.

\section{Funding}

OB is supported by a Monash University Postgraduate Research Scholarship and a Baker Bright Sparks Scholarship. J.I.M is supported by an Australian Government Research Training Program (RTP) Scholarship and Monash Graduate Excellence Scholarship. JES is supported by a National Health and Medical Research Council Investigator Grant and DJM by a National Health and Medical Research Council Senior Research Fellowship. This work is partially supported by the Victorian Government's OIS Program.

\section{Competing interests}

The authors declare no competing interests.

Additional information

Supplementary Information The online version contains supplementary material available at https://doi.org/ 10.1038/s41598-021-94099-3.

Correspondence and requests for materials should be addressed to O.B.

Reprints and permissions information is available at www.nature.com/reprints.

Publisher's note Springer Nature remains neutral with regard to jurisdictional claims in published maps and institutional affiliations.

\begin{abstract}
Open Access This article is licensed under a Creative Commons Attribution 4.0 International License, which permits use, sharing, adaptation, distribution and reproduction in any medium or format, as long as you give appropriate credit to the original author(s) and the source, provide a link to the Creative Commons licence, and indicate if changes were made. The images or other third party material in this article are included in the article's Creative Commons licence, unless indicated otherwise in a credit line to the material. If material is not included in the article's Creative Commons licence and your intended use is not permitted by statutory regulation or exceeds the permitted use, you will need to obtain permission directly from the copyright holder. To view a copy of this licence, visit http://creativecommons.org/licenses/by/4.0/.
\end{abstract}

(c) The Author(s) 2021 